\title{
ROTURAS UTERINAS
}

\author{
Dr. Rafael Ramírez Merchán* \\ Dra. Laura Rojas**
}

La coincidencia de dos artículos recientes (1-2) sobre rotura uterina con una revisión nuestra sobre el mismo tema (años 1963 a 1967), nos permite presentar estos comentarios en los cuales analizamos objetivamente el accidente y hacemos comparación de datos y resultados, lo cual le dá un interés y movilidad a las cifras estadísticas.

Incidencia. Revisamos un total de 125.673 partos correspondientes a los años 1963 a 1967 inclusive, del Instituto Materno Infantil de Bogotá. Encontramos ochenta roturas uterinas lo que dá una incidencia global de una rotura por 1.666 partos. El Medical College of Virginia sobre 112.637 partos revisados de 1957 a 1966 y dá 56 roturas con una incidencia de una por 2.011 partos. El Hospital Civil de Maracay registra 44 roturas uterinas sobre 34.801 partos del período correspondiente a los años de 1955 a 1965, con una incidencia para la rotura uterina, de una por 790 partos. Con este simple parangón se ve la disparidad en la incidencia que depende de múltiples circunstancias y sirve también para calificar la oportunidad y la calidad de los servicios prestados. Para referirnos a nuestra estadística, si le dedujéramos los casos en que la paciente ingresó con la matriz rota, tendríamos una incidencia de uno por 2.299 partos, inferior a la del importante centro americano. No pretendemos insinuar que estamos haciendo una atención hospitalaria ejemplar; estos índices, en un hospital universitario, debiera reducirse a la rotura extrahospitalaria que tiene causas muy complejas y ajenas a las funciones de nuestra Institución. (Cuadro No 1).

\section{CUADRO N: 1}

\section{ROTURAS UTERINAS - INCIDENCIA}

\begin{tabular}{lccr}
\hline Institución & No de partos & No de rupturas & Incidencia \\
\hline Med. Cent. Virg. 1957-1966 & 112.637 & 56 & $1: 2.011$ \\
H. Civ. Maracay. 1955-1965 & 34.801 & 44 & $1: 790$ \\
Inst. M. I. Bog. 1963-1967 & 125.673 & 80 & $1: 1.666$ \\
* Inst. M. I. Bog. 1963-1967 & 125.673 & 59 & $1: 2.299$ \\
* Descontando las extra-hospitalarias. & & & \\
\hline
\end{tabular}

* Profesor Titular del Departamento de Obstetricia y Ginecología. Facultad de Medicina. Universidad Nacional. Bogotá.
** Instructora. Departamento de Obstetricia y Ginecología. Facultad de Medicina. Universidad Nacional. Bogotá. 


\title{
CUADRO NN: 2
}

\author{
ROTURAS UTERINAS - ETIOLOGIA \\ NARCISO LOPEZ RISSO H. C. MARACAY. 1955-1965
}

\begin{tabular}{lr|ll}
\hline & & Fórceps & 8 \\
& & Versión & 6 \\
Total & 44 & Embriotomía & 2 \\
Espontáneas & 18 & Extr. Pod. & 1 \\
Traumáticas & 21 & Red de cordón & 1 \\
Desuniones & 5 & Extr. de hombro & 1 \\
& & Acc. Automov. & 1 \\
& & Fórceps versión & 1 \\
\hline
\end{tabular}

\section{CUADRO № 3}

ROTURAS UTERINAS - ETIOLOGIA

HERBERT A. CLAIBORNE. - ERIC SCHELIN. M. C. V. $1957-66$

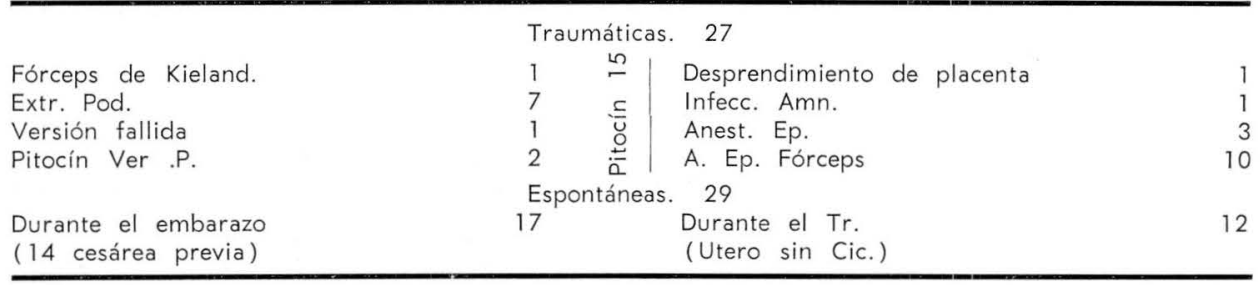

\section{CUADRO № 4}

ROTURAS UTERINAS - I.M.I. 1963-1967 ETIOLOGIA INTRA-HOSPITALARIA

\section{Traumáticas 16}

Prolong. herida Quir.

Versión por Ms. Int.

Fórceps O. D. P.

Ventosa O. I.

Pitocín O. I.

Maniobras en pelvis

Pitocín en mola $\mathrm{H}$.

(de 25 semanas)

Espontáneas? 43

Cesárea anterior

Vértice

Hombro

Abruptio placentae

Pres. de cara

Pres. de frente

Cicatriz corporal

Etiología. Roturas traumáticas. En los Cuadros Nos. 2, 3, 4 y 5, vemos las causas comparadas del accidente. Coinciden las tres instituciones en la clasificación, universalmente aceptada, de roturas espontáneas y traumáticas. Vale la pena anotar cómo va tomando trascendencia enorme en la producción de este accidente el uso de los ocitócicos; de los 56 casos del informe de Richmond en 15, la causa fue el Pitocín y en los del Instituto Materno-Infantil lo fué en cinco. No figura esta etiología en el informe de Maracay. Tenemos que advertir también que la causa más frecuente del accidente en pacientes que ingresan al Hospital con el útero ro-

\section{CUADRO NN 5}

\section{ETIOLOGIA EXTRA-HOSPITALARIA}

$\begin{array}{ll}\text { Antecedente de cesárea } & 5 \\ \text { Con abuso de Pitocín } & 5 \\ \text { Con "presentación" de hombro } & 4 \\ \text { Sin diagnóstico de pres. } & 3 \\ \text { Con retención de cabeza última } & 2 \\ \text { Cicatriz de miomectomía } & 1 \\ \text { Maniobras abortivas emb. de } 6 \text { meses } & 1\end{array}$


to 0 en inminencia de rotura, es el uso indebido de ocitócicos por comadronas empíricas. Más aún, tenemos bases para sospechar que en muchos de los casos que figuran en la estadística nuestra como rotura espontánea, hubo también abuso de ocitócicos antes del ingreso al Instituto. Por csa razón ponemos un interrogante en el cuadro de las roturas espontáneas. Nunca se insistirá lo suficiente en el rigor que debe presidir el empleo de los ocitócicos. Su indicación debe ser atributo de personal muy calificado y la vigilancia estricta de la paciente debe quedar en manos de personal idóneo. Tenemos un caso (Cuadro № 4) en que la rotura se produjo durante el trabajo inducido para la expulsión de una mola de 23 semanas; lo que demuestra el real peligro de la acción farmacodinámica de la droga en oposición a su efecto fisiológico precisado por Alvarez y Caldeiro (3). Las instituciones docentes, que deben dar la pauta, han disminuído el accidente al prescindir de intervenciones tales como fórceps alto, o medio alto, extracción podálica, versión por maniobras internas, maniobras de conversión y valoración juiciosa e individual de la posibilidad del parto por vía vaginal en las pacientes con antecedente de cesárea así como con la vigilancia permanente del trabajo y con la prescindencia sistemática del uso de ocitócicos en tales casos. En nuestro informe el fórceps no figura sino una sola vez en la etiología del accidente mientras que en el americano lo emplearon once veces y ocho, en el informe venezolano. La versión por maniobras internas figura una sola vez en nuestro informe; tres veces en el americano y seis en el venezolano. No tenemos nosotros casos de extracción podálica, maniobra que ha sido eliminada, mientras se repite siete veces en el informe americano y una en el de Maracay. Comentario aparte merece la rotura uterina del trabajo con anestesia peridural o caudal presente trece veces en la institución americana. En nuestras estadísticas no figura porque carecemos de servicio sistemático de anestesia para la generalidad de los partos y solamente se utiliza en contados casos; pero debemos dar la voz de alarma ante la posibilidad de la rotura uterina en pacientes que trabajan con engañosa normalidad por la acción de la anestesia regional, desde luego contraindicada cuando hay antecedente de cesárea y distocia de proporción o cuando no hay personal hábil que vigile el caso.

Está gravada nuestra estadística con cinco casos de "maniobras en presentación de pelvis". En tres de estos casos las pacientes ingresaron con feto muerto y retención de cabeza última. Debido a la grave amenaza que se cierne sobre el feto en el parto en presentaciones de pelvis, elocuentemente enumeradas Dougald Bayrd (4). En nuestra institución hemos definido como obligatoria la cesárea en las primigestantes con esta presentación, en paciente con antecedente de cesárea, en el parto gemelar con el primer gemelo en pelvis $y$ en las multigestantes en que se presente la menor distocia ya sea dinámica, con especial referencia al comportamiento cervical, o por falta de encajamiento, prolongación del trabajo, rotura prematura de membranas, y desde luego, en las distocias que de por sí exigen la intervención quirúrgica.

La ventosa obstétrica figura en dos casos de nuestra estadística; aunque el mismo Mallmstrom (5) le dá más amplitud de acción que al fórceps, entre nosotros tiene muy escasas simpatías $y$, en los casos mencionados, 
se aplicó en inminencia de ruptura lo que la contraindicaba formalmente.

Roturas espontáneas. Llama la atención la falta de detalles en cuanto a las condiciones en que se produjeron; en el trabajo de Richmond figuran doce casos de rotura espontánea durante el trabajo con útero sin cicatriz, de donde se deduce que se trataba de distocias descuidadas. Hay también 17 casos de rotura durante el embarazo, 14 de las cuales corresponden a cesárea previa. En nuestra estadística, las roturas espontáneas en su gran mayoría, (treinta sobre treinta y cuatro) corresponden a pacientes con antecedentes de cesárea y aun más asimilable al mismo renglón, por antecedente de miomectomía corporal. Las demás, ocurren en presentaciones distócicas: una de frente, cuatro de hombro, una de cara y cinco de vértice. Con razón, en la mayoría de los centros obstétricos americanos anota Helman (6) hay tendencia a resolver todo caso de parto de cesárea previa con otra cesárea. Una observación que salta a la vista en nuestros casos, es que en las pacientes de baja paridad la causa preponderante de la rotura la dá el antecedente de cesárea y en las multíparas, el mal uso de ocitócicos. En la revisión estadística y comentarios de Gabriel Acuña (7-8) solo el $40 \%$ de las pacientes con antecedente de cesárea tuvieron el parto por la vía vaginal; en éstas, hubo un $2 \%$ de rotura. Alberto Cárdenas Escobar (9) encuentra en su revisión sobre 100 casos de la Clínica David Restrepo, un $60 \%$ de partos por vía vaginal con un $1 \%$ de rotura uterina. López Risso (2) destaca cómo la mayoría de las estadísticas, y también la suya, informan que la multiparidad es factor frecuente en la etiología del accidente; agrega que la causa de la rotura de su estudio fué la desproporción cefalopélvica y a juzgar por el peso de los fetos: Caliborne y Schelin (1) (Cuadro № 3) sobre un total de 56, dan 29 de roturas espontáneas de las cuales 17 se produjeron durante el embarazo en la siguiente forma: con antecedente de cesárea, 14; y tres con útero sin cicatriz alguna. Pensamos nosotros que, como aún practican los estadinenses la cesárea corporal, esto explica la mayoría de las roturas durante el embarazo. La ocurrencia de cuatro casos de rotura durante el embarazo en útero sin cicatriz, corresponde a una patología inexistente en nuestras observaciones. No registramos ningún caso de rotura espontánea durante el embarazo. Las roturas que estos autores refieren durante el trabajo, son de útero sin cicatriz. Se ve claramente que la atención de las pacientes con antecedente de cesárea es tan cuidadoso, que en ellas no ha ocurrido un solo caso de rotura; falta saber si este Hospital practica la máxima "una vez cesárea, siempre cesárea"; lo que explicaría el cero de los accidentes. Al comentar el diagnóstico veremos que estos autores lo hacen antes de que se produzca, por la sensibilidad exquisita que manifiesta la paciente en la región suprapúbica relacionada con la cicatriz. Es claro que en estos casos la intervención logra los mejores resultados tanto maternos como fetales.

Edad y paridad. (Cuadros Nos. 6 y 7 ). Van paralelas como factor de importancia en la génesis de la ruptura como puede observarse en las gráficas. El más alto porcentaje lo dan las grandes multíparas, de G4 hasta G15, en nuestras estadísticas; la edad de mayor frecuencia es de los 31 a los 40 años. Es notorio en el informe estadinense la ocurrencia 


\section{CUADRO NN 6}

EDADES

\begin{tabular}{lllllr}
\hline 16 & años & & & 1 \\
De & 20 & a & 25 & años & 13 \\
De & 26 & a & 30 & años & 23 \\
De & 31 & a & 40 & años & 37 \\
De & 41 & a & 46 & años & 6 \\
\hline
\end{tabular}

\section{CUADRO NN 7}

ROTURAS UTERINAS - I.M.I. 1963-1967 PARIDADES

\begin{tabular}{lllrr}
\hline G2. & A1. & & & 1 \\
G2. & P1. & & & 7 \\
G3. & P2. & & & 4 \\
G4. & A G15. & & 68 \\
& & G13. & 5 & \\
\hline
\end{tabular}

de ruptura traumática en cinco primigrávidas en las cuales dos se produjeron por ocitócicos bajo anestesia epidural. En el IMI. de Bogotá no se registra ninguna primigrávida para el accidente $y$, en $G 2$, una traumática por maniobras abortivas para un embarazo avanzado. Roturas espontáneas en secundigestante hubo 7 casos, todas con antecedente de cesárea. Registramos, en cambio, $68 \mathrm{ca}$ sos sobre los 80 en grandes multíparas.

\section{CUADRO NN 8}

ROTURAS UTERINAS - I.M.I. 1963-1967 TIPO DE ROTURA

1) Cara anterior

\section{Segmentaria}

2) Cara posterior

3) Complicadas

4) Cicatriz cesárea

5) Dehiscencia

6) Prol. Inc. Quir.

\section{No Segmentarias}

1) Cicatriz corporal

2) Cicatriz miomect.

3) Fondo uterino

4) Bordes uterinos

Tipo de rotura. El Cuadro No 8 revela los tipos de rotura encontramos en el IMI de Bogotá. Por cuanto entre nosotros la cesárea de rigor es la segmentaria, la mayor frecuencia de roturas se observan en el propio segmento. En útero sin cicatriz, la mayoría de las veces, el segmento inicia la rotura. Como casos complicados se mencionan aquellos en que ha habido rotura de vejiga o rotura de segmento y cuerpo, o que ha sido interesada la arteria uterina.

Oportunidad del diagnóstico. (Cuadro $\mathrm{N}$ : 9). El diagnóstico prequirúrgico del cuadro se refiere a la oportunidad del mismo una vez producido el accidente y es el que dá los mejores resultados desde el punto de vista materno y algunos casos de supervivencia fetal. Tenemos un alto porcentaje de diagnóstico durante el acto operatorio en pacientes intervenidas por inminencia de rotura; es posible que el accidente se haya producido en el lapso transcurrido de la decisión operatoria a su realización. El diagnóstico tardío hecho en el puerperio inmediato o más tarde, revela inexperiencia del personal que atendió los casos o descuido, o apresuramiento explicable por la aglomeración de pacientes sumada a la escasez de recursos de que se dispone, pero que, en manera alguna justifica su ocurrencia. Una asistencia obstétrica ejemplar no tendría que hacer estos diagnósticos sino clasificar, valorar y vigilar cuidadosamente toda paciente en trabajo de parto.

Tratamiento. (Cuadro № 10). "La finalidad en sí del tratamiento, dice

\section{CUADRO N: 9}

\section{ROTURAS UTERINAS - I.M.I. 1963-1967 OPORTUNIDAD DIAGN.}

Pre-quirúrgico 22

Quirúrgico

Puerperio inmediato

Puerperio tardío 
Meredith (10) es la vida de la paciente $y$ no necesariamente hacer una completa disección quirúrgica". Tal ha sido también la pauta en el $|M|$ de Bogotá pero se tienen en cuenta paridad y edad; de ahí que en los, afortunadamente pocos casos, que se han presentado en secundigestantes se practicó cirugía conservadora con un ciento por ciento de buenos resultados. En las grandes multíparas en quienes se practicó cirugía conservadora, fue por imposición de las condiciones físicas de la paciente; en los casos que lo permiten se practica la histerectomía total. Prácticamente hay un $50 \%$ de histerectomías y un $50 \%$ de histerorrafias. Los resultados maternos finales son similares en los dos procedimientos. Los dos últimos renglones del cuadro corresponden a los casos desesperados en los que nada pudo hacerse; revelan casi en su totalidad la mortalidad materna. Decimos casi en su totalidad, pues hubo una muerta más, después de histerectomía total en paciente que ingresó en malas condiciones y con una rotura complicada.

\section{CUADRO № 10}

\section{ROTURAS UTERINAS - I.M.I. 1963-1967 TRATAMIENTO}

Histerectomía total

Histerectomía sub-total

Histerorafias

Laparotomías

No intervención
Mortalidad. (Cuadro № 11 ). Muestra el cuadro las cifras comparativas de las tres instituciones en cuanto a mortalidad materna y mortalidad perinatal. En cuanto a la primera, el Hospital de Maracay dá un 27,2\%, el Hospital de Virginia dá un 7,14\% y el IMI de Bogotá, un 7,50\%. Este último índice sería inferior si nuestra estadística no se recargara con los casos en que la paciente ingresa después de producida la rotura. $Y$, uno de los factores adversos, ha sido el diagnóstico tardío, pues cuando la intervención ha sido oportuna, no ha habido mortalidad. En relación con la mortalidad perinatal, entre el ciento por ciento de Maracay y el $37 \%$ de Virginia, Bogotá da un $63,7 \%$; pero vale la pena explicar que la supervivencia fetal no solamente depende de la oportunidad del diagnóstico sino también de la clase de rotura; en la rotura completa, la muerte fetal se produce en un ciento por ciento; en la dehiscencia y en las roturas incompletas, se logra supervivencia fetal en alto porcentaje. La notoria supervivencia fetal obtenida en el Medical Center se debe a los diagnósticos de roturas durante el embarazo pues registra cinco casos de rotura con antecedente de cesárea corporal en los cuales logran cuatro fetos vivos; y nueve casos con antecedente de cesárea segmentaria, todos con supervivencia fetal. A su vez $y$, para que se vea el contraste, presentan tres casos de rotura espon-

\section{CUADRO № 11}

MORTALIDAD

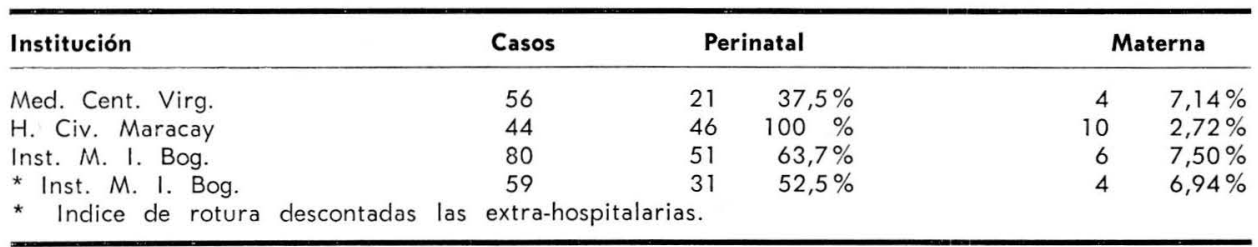


tánea durante el embarazo en útero sin antecedente de cicatriz, todos tres con muerte fetal.

Para finalizar estos comentarios insistimos en que el accidente, en nuestro medio, se reduciría al mínimo si se siguieran rigurosamente las normas y conductas establecidas, porque un alto porcentaje es imputable a la transgresión de tales normas. Es lo sucedido con el empleo de ocitócicos en pacientes con antecedente de cesárea y en las grandes multíparas; también al permitir parto vaginal a pacientes con antecedente de dos cesáreas o al practicar intervenciones vaginales a pacientes con inminencia de rotura.

Aun cuando hoy pueda parecer exagerado, vamos llegando al momento en que la cesárea sea de rigor, como en las indicaciones universalmente aceptadas, en las siguientes que aun provocan controversia:

1०) siempre que haya sido practicada una cesárea o que el útero grávido tenga cicatriz quirúrgica.

$2^{\circ}$ ) en todo caso de presentación de pelvis.

3) en las grandes multíparas con distocia dinámica por exceso o por defecto.

$4^{\circ}$ ) en pacientes con rotura prematura de membranas que, después de seis horas de trabajo iniciado oportunamente, no dejen la impresión clara de su pronta terminacijón espontánea.

\section{BIBLIOGRAFIA}

1 HERBERT A. CLAIBORNE Jr. ERICK SCHELIN. Am. J. Obst. and Gynec. 99-77. Dic. 1967.

2 NARCISO LOPEZ RISSO. Rev. Obst. Gin. Venezuela. 27. 645. 1967.

3 HERMOGENES ALVAREZ Y ROBERTO CALDEYRO BARCIA. Fisiopatología de la contracción uterina. Relato oficial presentado al II Congreso Latino-americano de Cbstetricia y Ginecología.

4 DUGALD BAIRD. Combined Textbook of Obstetrics and Gynecology. Fifth Edition.

5 TAGE MALMSTROM and INGE JANSSON. Use of the vacuum extractor Clinical Obstetrics and Gynecology. Vol. 8 № 4 Dic. 1965.

6 EASTMAN - HELMAN - WILLIAMS. Obstetrics. 13a. edition.

7 GABRIEL ACUÑA. El parto vaginal en paciente con antecedente de cesárea. Rev. Colombiana de Obst. y Gin.

8 GABRIEL ACUÑA. Porvenir obstétrico de la paciente con antecedente de cesárea. Trabajo presentado en el $\vee$ Congreso Nacional de Obstetricia y Ginecología. Cartagena.

9 CARDENAS E. ALBERTO. La Cesárea iterativa. Revista de la Clínica de Maternidad David Restrepo. Vol. 3 № 1.

10 RICHARD MEREDITH. Rupture of the uterus. Clin. Obst. and Gyn. III. 1960. 